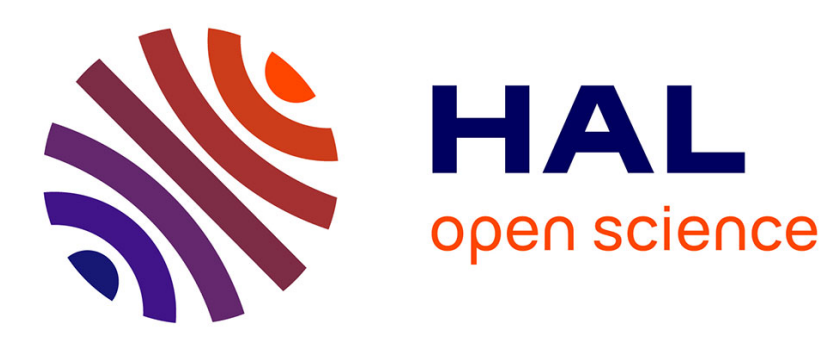

\title{
Comparison of some end-to-end flow control policies in a packet switching network
}

Guy Pujolle

\section{To cite this version:}

Guy Pujolle. Comparison of some end-to-end flow control policies in a packet switching network. [Research Report] RR-0001, INRIA. 1980. inria-00076560

\section{HAL Id: inria-00076560 \\ https://hal.inria.fr/inria-00076560}

Submitted on 24 May 2006

HAL is a multi-disciplinary open access archive for the deposit and dissemination of scientific research documents, whether they are published or not. The documents may come from teaching and research institutions in France or abroad, or from public or private research centers.
L'archive ouverte pluridisciplinaire HAL, est destinée au dépôt et à la diffusion de documents scientifiques de niveau recherche, publiés ou non, émanant des établissements d'enseignement et de recherche français ou étrangers, des laboratoires publics ou privés. 


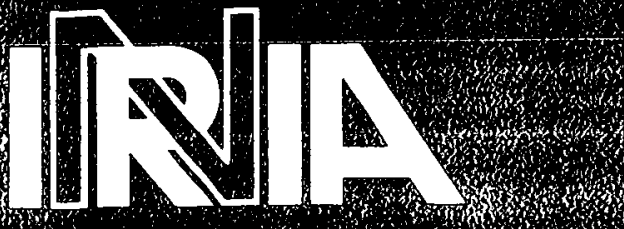

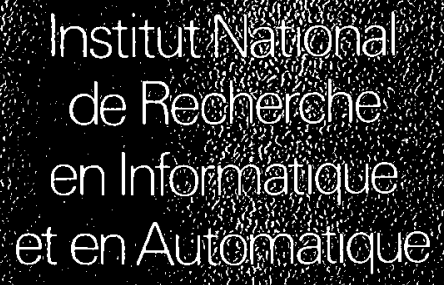

Domaine aevoluceau Rocqueneourt

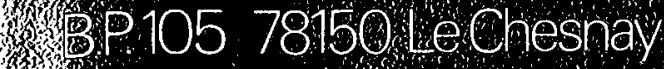
France

Tél.95 9020

\section{Rapports de Recherche}

\section{COMPARISON OF SOME END-TO-END FLOW CONTROL POLICIES IN A PACKET SWITCHING NETWORK}

\section{Guy PUJOLLE}

Janvier 1980 


\title{
IN A PACKET SWITCHING NETWORK
}

\author{
GuY PUJOLLE \\ IRIA-LABORIA \\ 78150 Le Chesnay \\ France
}

\section{Abstract}

In this work a model of packet switched networks is developed which reflects three possible flow control policies : a window flow control, a rate flow control and a flow control suggested by the X25 recommendation. The model is used to compare the throughput allowed by these different schemes.

\section{Résumé}

Dans ce rapport, nous développons un modèle de réseau à commutation de paquets permettant de tenir compte de trois politiques distinctes de contrôle du flux : un contrôle par fenêtre, un contrôle sur le débit d'entrée, un des contrôles possibles induit par la norme X.25. Le modèle est utilisé pour comparer les débits obtenus par les différentes politiques de contrôle. 


\section{1 - Introduction}

Various techniques may be considered when it comes to setting up a communications system between computers. The "packet switching" technique described by Davies [1], seems to be one of the best existing approaches. In the following we consider only such a technique : users of a computer network communicate with each other by mean of an intervening store-and-forward packet switching network.

The term host has been introduced in the Arpanet literature. It has been used widely, although not always in the very same sense as in Arpanet. We use it here is some loose sense : a host is a source and a sink of packets. A subscriber is an entity which provides to the host the data to be transmitted through the packet switching network (PSN). The set of subscribers attached to hast $i$ asks on the average the transmission of $\lambda_{i}$ packets per second.

It is well known in a system where resources are shared that when the load increases, it is necessary to have a congestion tool to avoid a degradation of performance. Such a phenomenon has been pointed out in PSN [2] [3]. Thus tools are necessary to prevent this degradation. They are flow control methods, namely, procedures whereby the receiver allocates a potential transmission credit to the sender, no matter what the form may be to specify this credit.

In section 2, we describe several types of specifications in order to compare them in section 5 . To do this, we shall introduce in section 4 a unified mathematical model. This model will use a single source destination path taking into account other arrivals at intermediate node. Two different node transmission procedures, introduced in section 3 , will be used in the model.

The main contribution of our paper is that we take explicitely into account most of the elements which characterize a packet switching network : node to node and host-to-host protocol, retransmission policy, and finite buffer size in nodes. Three flow controls are examined and their performance compared in detail under several working assumptions. 
We show that the maximum throughput allowed by these three types of flow controls are very different and that the higher the throughput, the more it is important to control adequately the parameters of the system to avoid a thrashing phenomenon.

\section{2 - FLOW-CONTROL TECHNIQUES}

\section{1 - Window flow control.}

One of the best known flow control techniques is the isarithmic scheme [1][2]. Under this control, there are a fixed number of credits circulating in the network. A packet is admitted into the network only if it can get hold of a free credit. The packet travels through the network accompanied by its credit. The credit is again free when the packet reaches its destination. Several policies can be followed to redistribute the free credits. For example they can be made available to any host in the network. They can be host-dedicated, in that they return to the originating source when they are released. Or they can be host-to-host dedicated, a scheme corresponding to a window flow control and the number of credits used for an host-to-host communication corresponds to the value of the window width. Our first flow control WFC (Window Flow Control) is exactly this last one. The size of the window, $w$, defines the maximum number of simultaneous outstanding (or unacknowledged) frames permitted between the two hosts. A window is closed and further transmission blocked when all w frames are outstanding.

\section{2 - Rate flow control}

An attractive stheme can work by a limitation of packets entering into the network in the following way. As long as a destination is able to cope with the outgoing packets, there is no need to choke the sending host-sources. However if there is an excess of traffic, queues will start building up, and will eventually block the nodes. It is convenient to let each host receive from each node the information of the maximum amount of packets it can accept. According to this knowledge, hosts can then limit their transmissions to a "good" number of packets per unit of time. 
In a first step we shall assume a fixed threshold on the number of packets that can enter the network each unit of time. Then this number will vary with the state of the network. We call this technique the rate flow control (RFC).

\section{3 - Flow control induced by the $\mathrm{X} 25$ recommendation}

CCITT Recommendation X25 [4] has been selected by some telecommunication organizations as one of their packet network user interfaces. This implies its importance. X25 specifies the protocols for exchanging information between similar levels in the Data Terminal Equipment (DTE) and Data Circuit Termination Equipment (DCE).

Three levels have been identified in the X25 Recommendations. Leve1 1 is the physical interface. Leve1 2 is the link access procedure. The use of level 2 is primarily for error control on an errorprone datalink. The virtual circuits, at level 3, should be comparatively error free and so the emphasis is on flow control. A virtual circuit has to be set up by an exchange of packets before information transfer can take place. An independent window fow control mechanism is used for each virtual circuit. But CCITT X25 Recommendations does not specify how the control information should be interpreted. This would depend on whether level 3 is implemented to perform end-to-end control or to perform local control. In this paper we shall assume X25 provides a end-to-end contro1. Moreover the sep up packet is in charge to reserve some resources when going accross a node.

So at the host leve1, the flow control induced by the X25 Recommendation, can be seen as a superposition of virtual circuits. Moreover we sha11 assume that the number of buffers reserved at each node by the set up packet is equal to the value of the window size. Thus, globally the flow control induced by the X25 Recommendation is based upon authorization from the network (sends by the receiver).

The authorization for a packet is given if at less one buffer is available in each node the packet has to be gone through. 
If overallocation of buffers is authorized for virtual circuits, new packets can enter the network until this limit is reached. We sha11 assume that virtual circuits establishment and release are negligible.

In the sequel we call XFC (flow control induced by X25) the control described above assuming no overallocation.

3 - SPECIFICATIONS OF THE PACKET SWITCHING NETWORK.

Before we develop the mathematical model, it is necessary to define some specifications of the packet switching network itself.

We must define a strategy for dealing with packets rejected because of overflow due to finite buffer size at the switching nodes. We shall take into account two cases :

1 - the most common technique, here called switch-retransmission (used for example in ARPA) in which if a packet cannot be accepted by a node, it is retransmitted from a backup copy held in the preceding node.

2 - Another technique which we call host-retransmission (used in the Cyclades network) in which the network drops a packet which arrives at a full switch, to be resent later by the source host.

Moreover, two types of node-to-node transmission procedures are allowed that we describe now.

3.1 - "Send and wait" procedure

Before transmitting a new packet, the previous one must be acknowledged. On the time axis of figure 1 we have represented the state of the sender and of the receiver during the transmission of a packet 


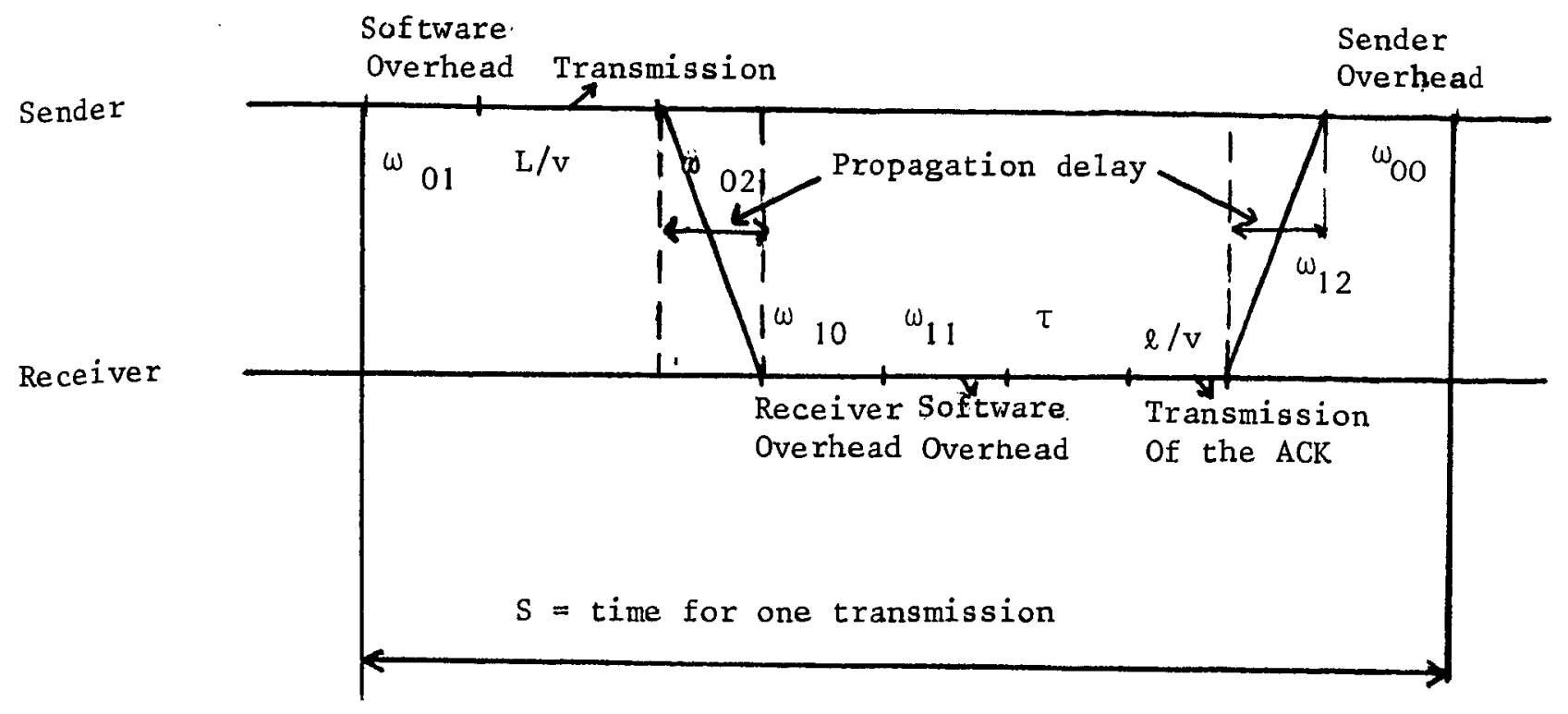

\section{The "send and wait" behavior}

\section{Figure 1.}

$\omega_{00}, \omega_{10}$ are due to the task switching and software work of the
packet.

$\omega_{01}, w_{11}$ are the software delay of the writing on both systems.

$\omega_{02}, \omega_{12}$ are due to the propagation delay, and depend on the modems used and the line length (overhead due to modems are not negligible).

- L is the mean total length of packets to be transmitted,

- $\ell$ is the length of the control packet which returns the acknowledgement (ACK).

- $v$ is the line capacity.

- $\tau$ represents the time that the previous packet if any takes to finish its transmission.

We denote by $S$ the total time necessary for the transmission of a packet. This mean service time is given by :

$$
S=\omega_{00}+\omega_{01}+\omega_{02}+\frac{L}{v}+\omega_{10}+\omega_{11}+\omega_{12}+\frac{l}{v}+\tau
$$


For the various overheads we will use the following values measured on the Cyclades network [5]

$$
\omega_{00}=\omega_{10} \simeq 5 \mathrm{~ms}, \quad \omega_{01}=\omega_{11} \simeq 3 \mathrm{~ms}
$$

For a $500 \mathrm{~km}$ line length $\omega_{02}=\omega_{12} \simeq 3 \mathrm{~ms}$.

We have to note the variation of $\tau$ according to the load on the lines. If the load is weak, $\tau=0$ almost surely. In heavy traffic on the average $\tau$ is the transmission time of half a packet length.

In the sequel, we assume the traffic is symmetric so that there are equal amounts of forward and return traffic. Modification of the result for nonsymmetric traffic is not difficult. We denote by $\rho$ the load on a line. If $\rho=0$ then the service $S$ is minimum and if $\rho=1$ the service time is maximum ${ }^{*}$. We shall adopt a linear variation of the mean service time $S$ between its maximum and its minimum.

Let $\mathrm{Ca}=\frac{1}{\mathrm{v}}$ and $\mathrm{Cb}=\omega_{\mathrm{OO}}+\omega_{10}+\omega_{01}+\omega_{11}+\omega_{02}+\omega_{12}+\frac{l}{\mathrm{v}}$. We obtain the following simple expression for $\mathrm{S}$ :

$$
\mathrm{S}=\mathrm{CaL}+\mathrm{Cb}+\mathrm{Ca} \frac{\mathrm{L}}{2} \rho \text {. }
$$

If the line speed is $48 \mathrm{~kb} / \mathrm{s}$, we have for a $500 \mathrm{~km}$ line length :

$$
\mathrm{S}=20.8 \mathrm{~L}+26+10.4 \mathrm{~L} \rho
$$

The quantity $S$ we have defined is the time necessary to transmit successfully one packet. Now if an error occurs during the transmission or if the packet is rejected by an overflow in the receiver node, a backup copy has to be transmitted after a time-out. We have shown in a previous paper [6] that the performance of the node-to-node procedure is not sensitive to the probability of packets in error for usual values of this probability. Thus we assume this probability negligible. Let $p$ be the probability of overflow of the receiver node.

If we use the switch-retransmission ( $\mathrm{s} r$ ), a backup copy is retransmitted after a time-out $\mathrm{T}$ with the probability $\mathrm{p}$. So the mean real time for one transmission is : (without the retransmission if the packet is lost).

* note : $\tau=0$ and $\tau$ is the transmission of half a packet length, respectively. 


$$
\mathrm{S}_{\mathbf{s r}}^{\mathrm{SW}}(\rho)=\left(\mathrm{CaL}+\mathrm{Cb}+\frac{\mathrm{CaL}}{2} \rho\right)(1-\mathrm{p})+\mathrm{T} \mathrm{P}
$$

We assume $\mathrm{T}=200 \mathrm{~ms}$ for a $48 \mathrm{~Kb} / \mathrm{s}$ line.

For the host-retransmission (hr) the overflow is detected after the acknowledgement is sent (the overflow is detected by the switch). So the mean time for one transmission is

$$
\mathrm{s}_{\mathrm{hr}}^{\mathrm{SW}}(\rho)=\mathrm{CaL}+\mathrm{Cb}+\frac{\mathrm{CaL}}{2} \rho .
$$

\section{$\underline{3.2-\text { HDLC procedure }}$}

The HDLC procedure (High Level Data Link Control) has been accepted as an international standard. Its behavior is shown in figure 2. A window is defined between the sender and the receiver. The size of the windowdefines the maximum number of simultaneous unacknowledged packets permitted between the two nodes.

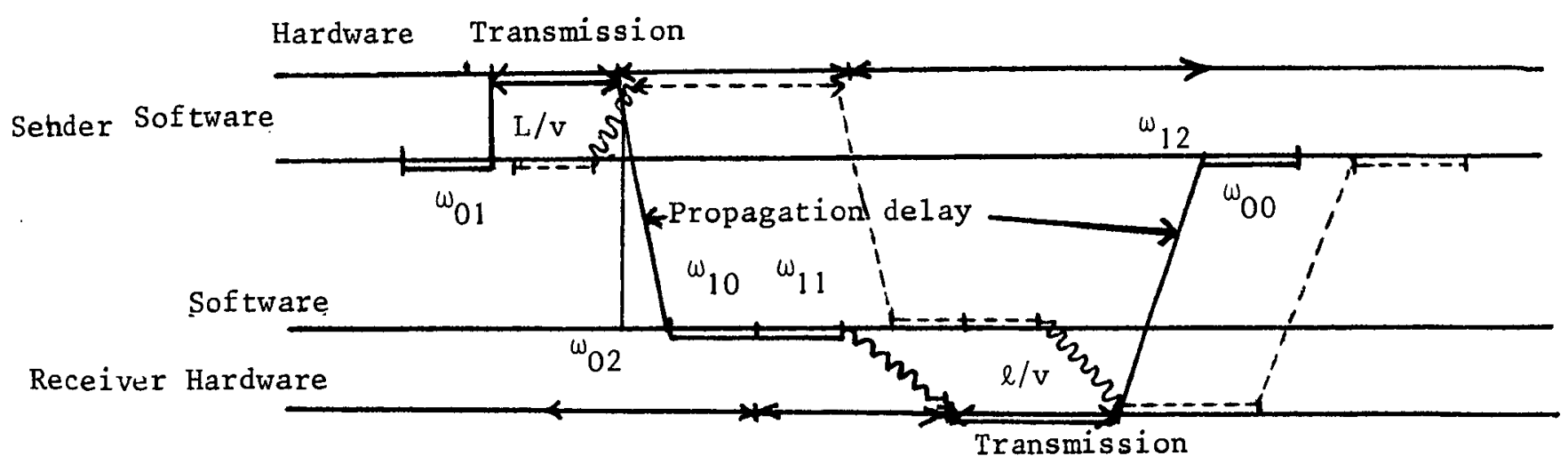

Behavior of the HDLC protoco1

\section{Figure 2}

Due to the parallelism of the processes, the effective time required for a transmission is difficult to calculate; it depends on the window width. However it is shown in [6] that the throughput is only limited by transmission times if the window width is chosen adequately (the window width has to be sufficiently large so that no blocking occurs). 
Thus we shall adopt for the mean effective thranmission time the following values: $S_{s r}^{\mathrm{HDLC}}(\rho)=\operatorname{CaL}(1-\mathrm{p})+\mathrm{Tp}$, or $S_{h r}^{\mathrm{HDLC}}(\rho)=\mathrm{CaL}$.

\section{4 - THE UNIFIED MATHEMATICAL MODEL}

Let the routing strategy for the network be fixed. Then the host transmits packets to some destination along a fixed path through the network consisting of a series of intermediate storage nodes connected by communication lines. In addition to the packets transmitted by the host, packets from other hosts also join the queues and obtain service. They are called external packets.

If we assume the network topology symmetrical, the number of external packets entering a node is equal to the number of external packets leaving the node after service. On the average, we can assume that it is a same packet which goes through the tandem queues. This assumption seems to be accurate due to our computation assumption which assume Poisson input for each queue.

Such a route is modelled as a tandem queueing network. The system consists of $\mathrm{K}+1$ queues. A customer (corresponding to a packet) goes through the system joining the $(i+1)$-th queue after the $i-$ th device for $0 \leq \mathrm{i} \leq \mathrm{K}-1$.

The first station 0 , corresponding to the host, is assumed to have an infinite number of buffers. We assume that one packet and only one can be contained in one buffer. All the other stations have a finite number $M_{i}$ of buffers (there is room for only $M_{i}$ packets at station $i$ ). This 
finite size corresponds to the storage facility of the transmission lines of the end-to-end route. Processing time within the switching nodes are considered through the procedure models. This mathematical model is shown in figure 3 . The dotted lines represent either the switch-retransmission technique or the host-retransmission technique.

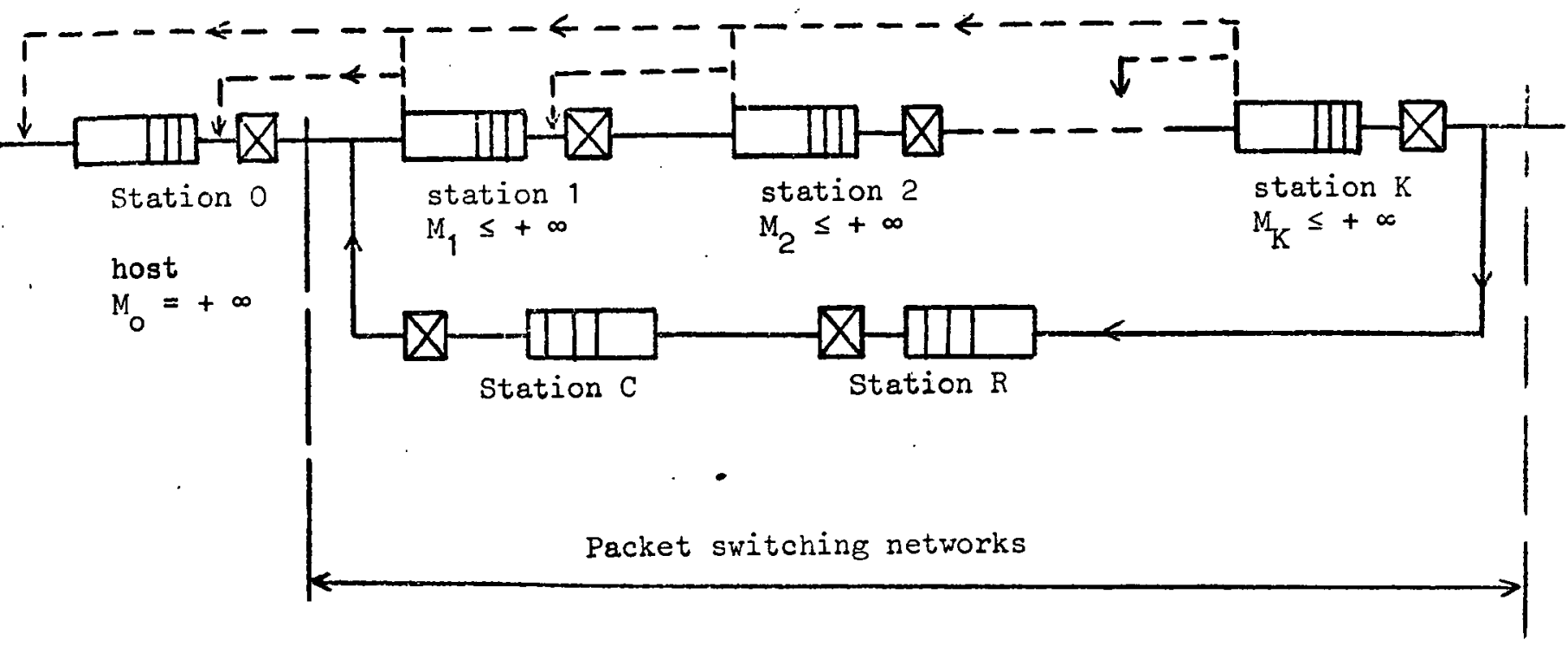

The unified mathematical model

\section{$\underline{\text { Figure } 3}$}

In figure 3 , customers of station $C$ represent the credits. If its queue is empty the host must wait for a credit before transmitting a packet. The number of credits in the packet-switching network is denoted by $\mathrm{N}$. When a packet leaves the network a credit comes towards the host. It passes through the station $\mathrm{R}$ which represents the return time of the ACK (containting the free credits). When a customer flows from station 0 to station 1 a credit disappears.

Our three flow control policies can be characterized as follows. 
In the window flow control, the total number of credits circulating in the end-to-end route represents the window width. We have to note that if this number $\mathrm{N}$ of credits is less than or equal to $\operatorname{Min}\left(M_{1}, M_{2}, \ldots, M_{k}\right)$, there is always a buffer available for a packet entering the network. This corresponds to the XFC technique.

If the total number of credits is greater than the sum $M_{1}+M_{2}+\ldots+M_{K}$ and assuming station $R$ does not exist, we have a network without flow control.

Finally the rate flow control policy will be studied at the same time as the case without flow control because it corresponds to a threshold on the utilization of the server of the host.

In the applications, the mean service time will be chosen to be one of $\mathrm{s}_{\mathrm{sr}}^{\mathrm{SW}}, \mathrm{s}_{\mathrm{sr}}^{\mathrm{HDLC}}, \mathrm{s}_{\mathrm{hr}}^{\mathrm{SW}}, \mathrm{s}_{\mathrm{hr}}^{\mathrm{HDLC}}$ according to the node-to-node protocol and retransmission strategy chosen. We shall denote by $S$ this mean service time when the choice between several policies is possible.

Solution of the unified mathematical model.

Several criteria can be used to evaluate the performance of the models. As we deal with flow control schemes we need an indicator of performance according to which the system is judged. This congestion measure can reasonably be chosen to be the throughput of the system versus the utilization of the server of the host, i.e. the transmission line between the sending host and the first switching node. We have chosen this last parameter because it allows to compare the different flow control sohemes under an unified manner, and it is one the parameters used to control the traffic entering the packet-switching network.

The solution of the unified mathematical model will be carried out in two steps :

1 - the model without station $C$ and $R$.

2 - the model with station $C$ and $R$.

In order to solve this complex model some simplifying assumptions must be made; we describe them now. 
In a real PSN each packet maintains its length as it travels from node to node, and service times are not independent. Here we will make the independence assumption of Kleinrock [7] and a new independent packet length will be chosen at each station.

We assume that the distribution of service times of all the stations are identical and the average value is $S(\rho)$ where $\rho$ is the utilization of the server of the host. This assume that all the stations have the same utilization rates. This is accurately verified in balanced networks.

Finally, we assume that a customer leaving a queue sees the system in an equilibrium state, namely, the probability for a packet to be rejected is taken equal to the probability that the following queue is full. It has been shown [8] that this assumption is quite accurate.

The explicit computations are introduced in the appendix. We just : give an idea here : from a given utilization of the host $\rho$, we compute the probability $p$ that a customer is rejected by the PSN and comes back into the host. Then the mean transmission time is obtained from $S(\rho)$ whose value depends on the node-to-node protocol and the retransmission policy. Therefore we obtain the total arrival rate $\lambda^{*}$ as $\lambda^{*}=\rho / S(\rho)$. This rate is the sum of external arrivals and recycling packets, therefore the throughput of the system will be : $\lambda=\lambda^{\star}(1-p)$.

Though computation can be carried out with a different buffer size at each node, we shall assume $M_{i}=M, i=1, \ldots, k$.

\section{5 - RESULTS AND COMPARISONS}

\section{1 - Results without flow control policy}

For the SW procedure, we have compared the two retransmission policies. On figure 4, some curves have been drawn representing the throughput versus the utilization of the host for both the situations of noderetransmission and host-retransmission, for a 48kbits/sec. line and 5 or 8 buffers at each output line, for 6 stations in series ( 1 host and 5 nociess).

It is important to notice (this is true for all the following results) that the maximum throughput is reached for an utilization of the server of the host equal 1. Namely a throughput greater than this value cannot 
Throughput

packets/sec.
Throughput

normalized

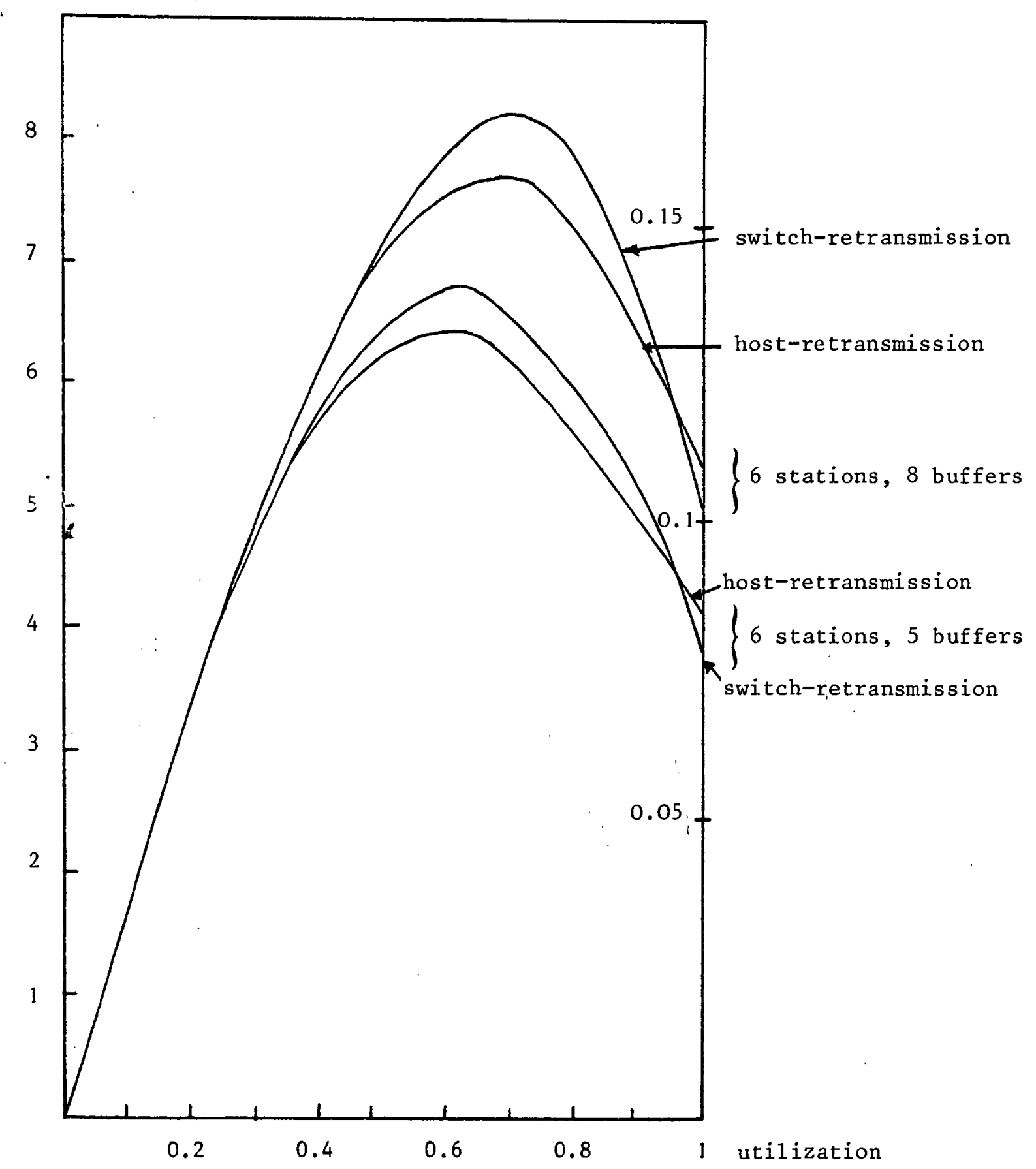

Throughput versus the utilization rate of the host

line capacity $=48 \mathrm{Kbits} / \mathrm{sec}$.

mean packet lenth $=1 \mathrm{Kbit}$

packet length distribution $=$ exponentia1

$$
\text { procedure }=\mathrm{SW}
$$

\section{Figure 4}


be reached without flow contro1. This implies that the points corresponding to higher throughput than for $\rho=1$ are unstable points.

Thus, we see in figure 4 , that without flow control host-retransmission leads to a better throughput than switch-retransmission. This can be easily explained : when we approach saturation $(\rho=1)$ the switchretransmission policy increases the congestion whereas the host-retransmission policy prevents congestion. This is even more explicit for the HDLC node-to-node procedure (see figure 5 ).

We notice by examining figure 4 and 5 , that if a flow control policy exists and allows us to obtain a throughput near the optimal point (the highest point of the curve) switch-retransmission is better than host-retransmission. This can also be easily explained : the optimal point is surely obtained when there are only few retransmissions but when the lines are utilized at the maximum. In this case to come back to the host is worse than to reset from the previous switch.

Since the purpose of this paper is to study and compare flow control methods, we limit ourselves to the switch-retransmission case, which is the best retransmission policy in this case.

We show in figures $6,7,8$ the differences provided by the change of service time distribution and mean packet length. We have adopted a $48 \mathrm{Kbits} / \mathrm{sec}$ line and 3 or 6 tations in series, 5, 8 or 12 buffers per output line, and SW or HDLC protocols.

The degradation predicted is very clear and more important with HDLC than with the SW procedure. As long as there is no degradation (namely the probability of retransmission is negligible) 3 or 6 stations in series give the same throughput. Degradation is evidently stronger for 6 stations than for 3 stations in series.

The degradation is so much important when mean packet length is short and packet lengths are irregular. These three figures give an idea of throughput that can be obtain between two hosts of a PSN. 


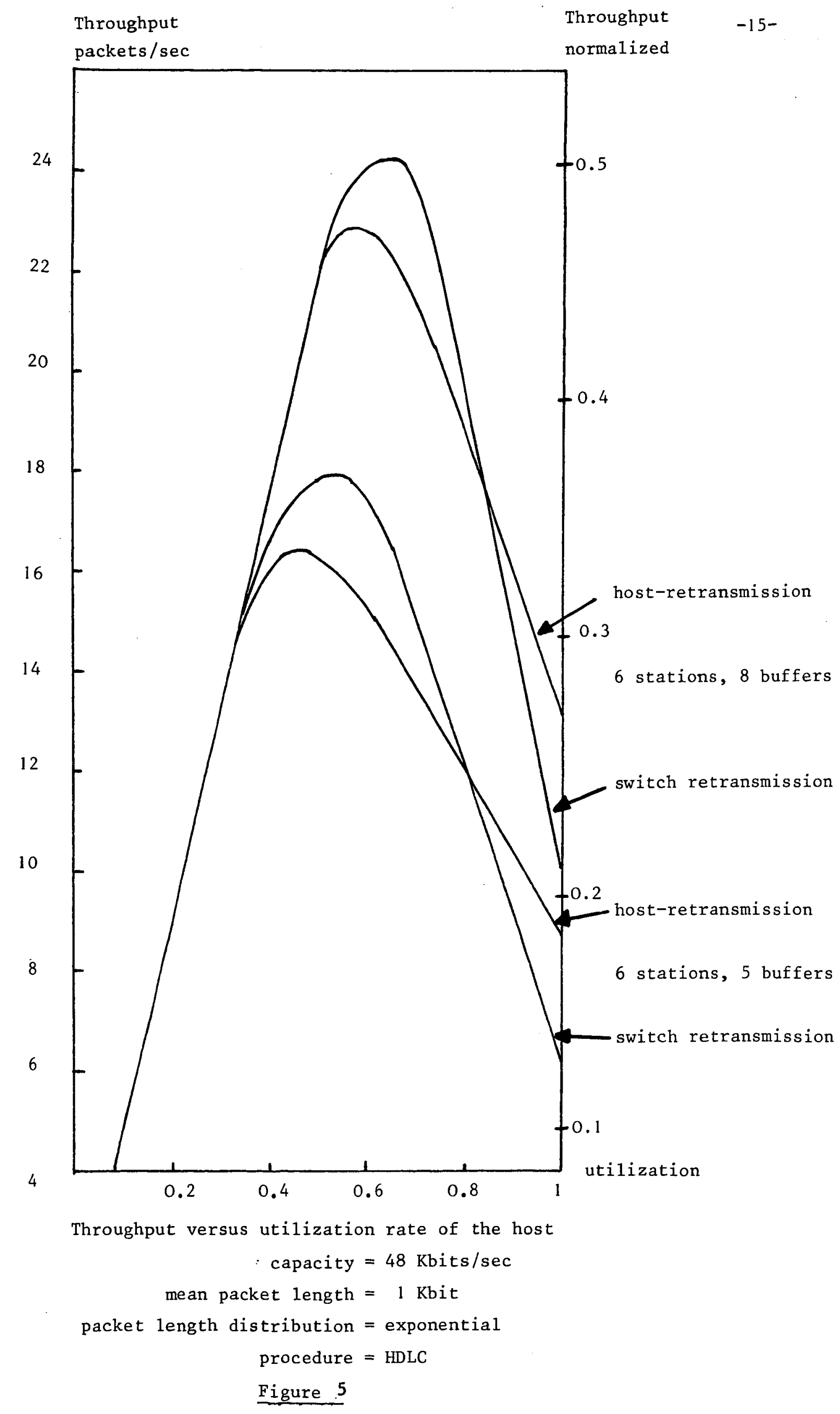


$-0.4$

3 stations, 12 buffers, HDLC

6 stations, 12 buffers, HDLC

3 stations, 8 buffers, HDLC 0.3 stations,

6 stations, 8 buffers, HDLC 0.2 $\$-0.2$

Throughput versus the utilization rate of the host line capacity $=48 \mathrm{Kbit} / \mathrm{sec}$ 


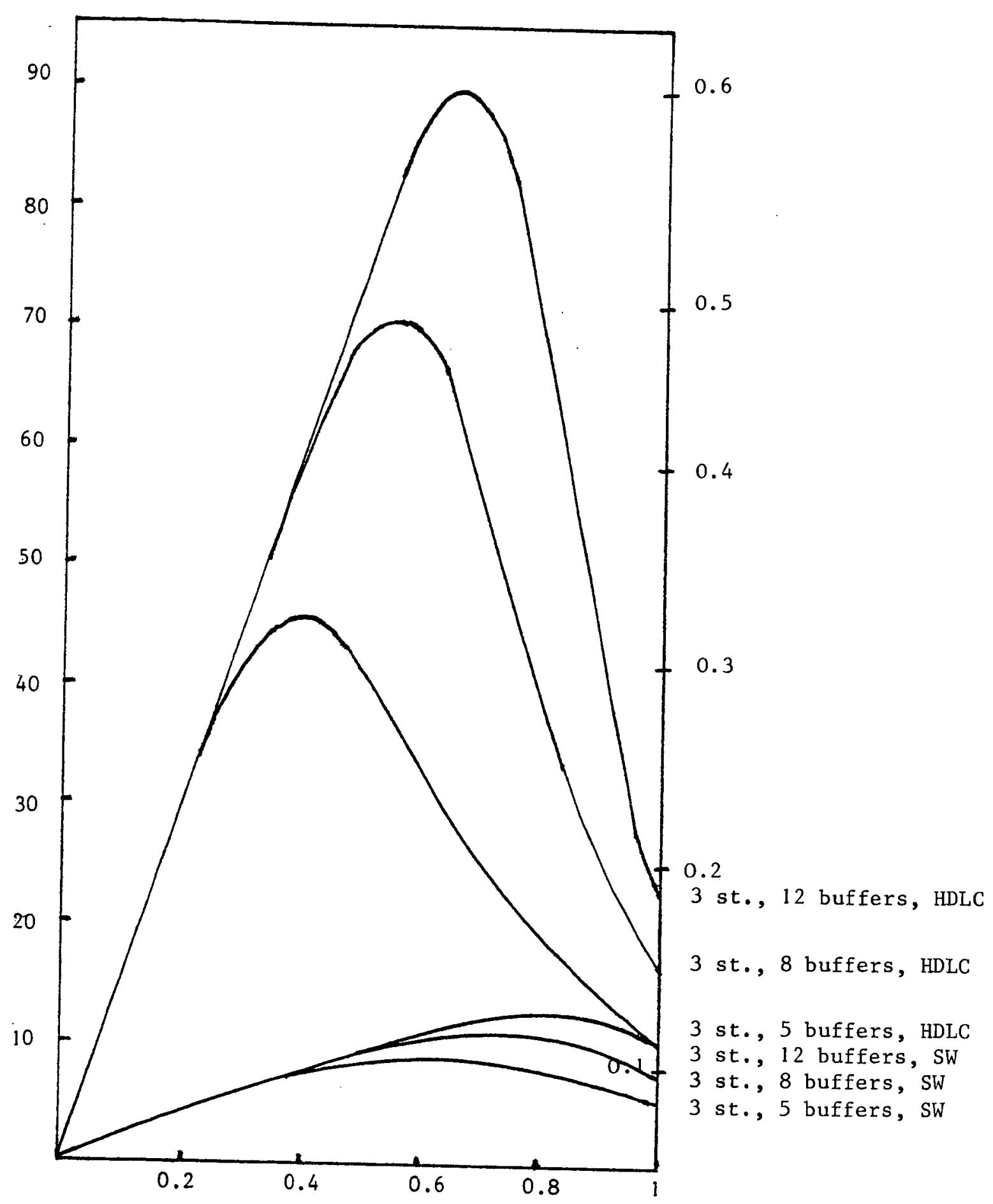

Throughput versus the utilization rate of the host

$$
\text { line } \text { capacity }=48 \mathrm{Kbits} / \mathrm{sec}
$$

mean packet length $=300 \mathrm{bits}$

packet length distribution = exponential

\section{Figure 7}


Throughput (packets/sec)

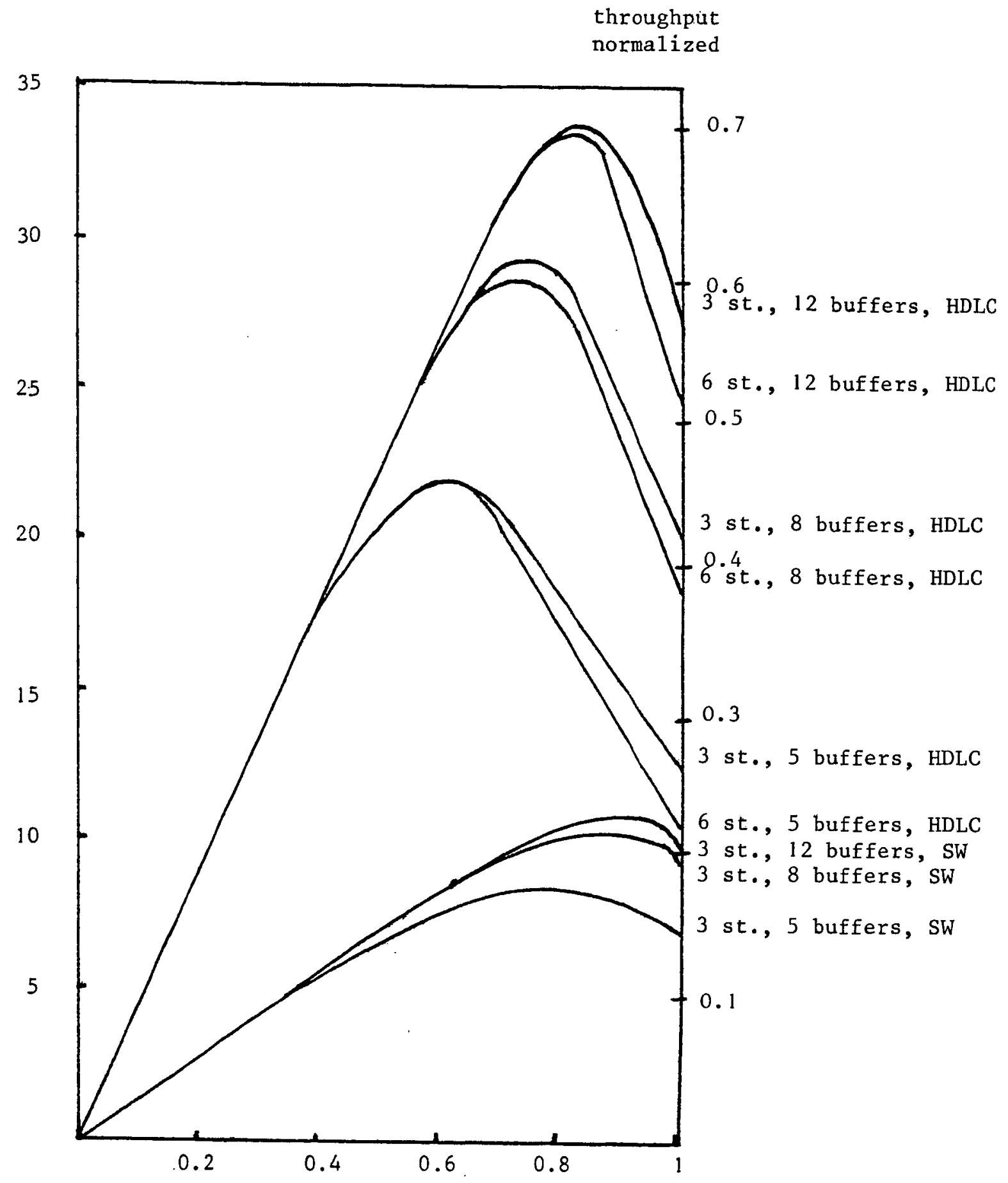

Throughput versus the utilization rate of the host

line capacity $=48 \mathrm{Kbits} / \mathrm{sec}$

mean packet length $=1 \mathrm{Kbit}$

packet length distribution $=$ Erlang 2

\section{Figure 8}




\section{2 - "Rate flow control policy (RFC)}

The rate flow-control policy using a threshold on the number of transmissions per unit time can be studied with the previous results. This threshold corresponds to a value $\rho^{\star}$ of the activity of the server of the host. In figures $8,9,10$ the maximum throughput is now obtained for the value of the throughput corresponding to $\rho^{*}$ and the parts of the curves on the right of $\rho^{*}$ cannot be reached.

It is obvious that the quality of this flow control policy depends on the choice of $\rho^{*}$. For the case studied in figures $6,7,8$ it is sufficient to take for $\rho^{*}$, the activity of the host corresponding to the optimal value of the throughput. However, in a general network, according to the destination of packets, the line capacities and the sizes of the pools of buffers, the optimal throughput does not correspond to the same 1imitation. Therefore, an efficient estimation and control system is necessary to adjust the threshold according to the state of the nodes of the PSN.

This control leads to high throughput, but the risk of a strong degradation of performance exists as soon as the system is badly controlled, (i.e. determination of a worse $\rho *$ ).

In Cyclades such a flow control is used and the rate defining the threshold varies according to the load of the lines of the system.

\section{$5.3-$ WFC and XFC policies}

In figures 9 and 10 the two other flow controls are examined using the solution of the second part of the appendix to solve the unified mathematical model. We look at the case of $48 \mathrm{kbits} / \mathrm{sec}$ line, 6 stations in series and 5 buffers per output line of nodes. Figure 9 corresponds to the SW node-to-node protocol and Figure 10. to HDLC. $N$ is the number of credits. If $\mathrm{N}=5$, we obtain the XFC. For example we have a superposition of five virtual circuits between the two hosts with a window width equal to 1 . If $\mathrm{N}=25$, we find again the case without flow control or with RFC if a $\rho^{\star}$ is given. Now if $5<\mathrm{N}<25$ we obtain a WFC. We assume that the queue $R$ of the mathematical model does not exist. 
Throughput packets/sec

Throughput normalized

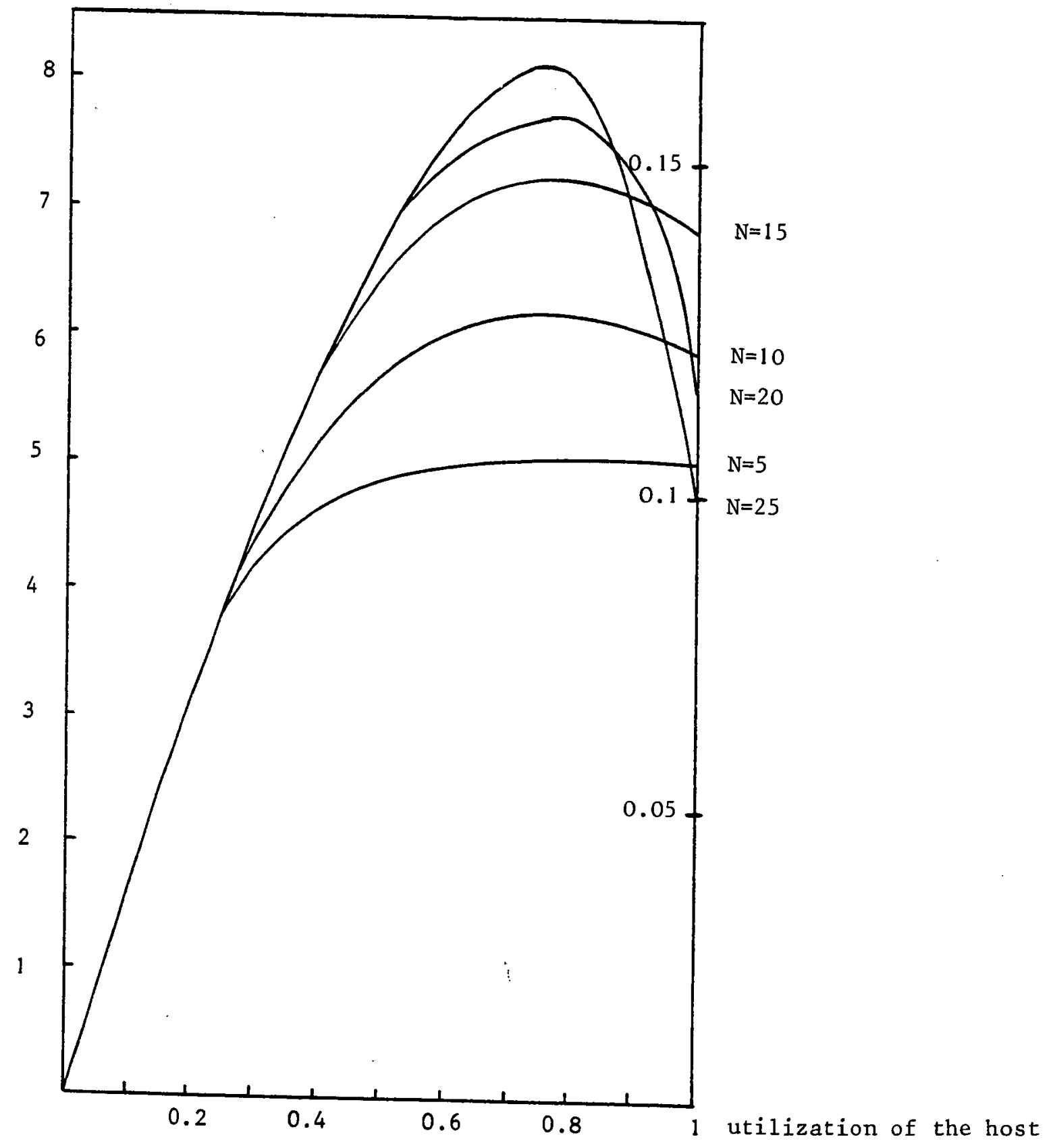

Throughput versus the utilization of the host, for a number of credits N, 6 stations in series and 5 buffers per station.

$$
\begin{aligned}
\text { line capacity } & =48 \mathrm{Kbits} / \mathrm{sec} \\
\text { mean packet length } & =1 \mathrm{Kbit} \\
\text { procedure } & =\mathrm{SW} \\
\text { packet length distribution } & =\text { exponential }
\end{aligned}
$$

Figure 9 


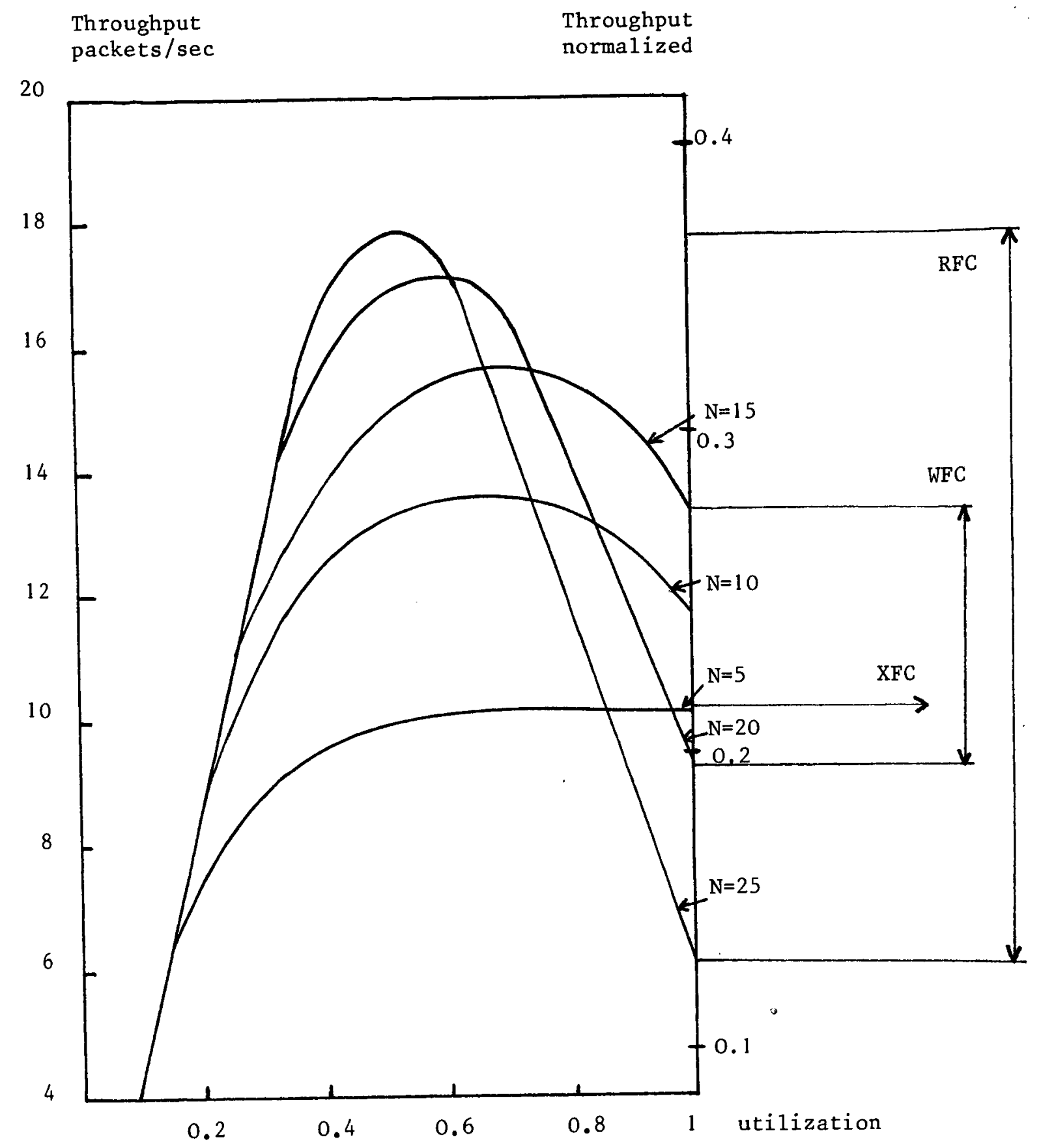

Throughput versus utilization of the host, for a number of credits $N$, 6 stations in series and 5 buffers per station.

line capacity $=48 \mathrm{Kbits} / \mathrm{sec}$

mean packet length $=1 \mathrm{Kbit}$

procedure $=$ HDLC

packet length distribution $=$ exponential

Figure 10 
The advantage of the XFC is that no thrashing phenomenon exists. Above a certain value of the activity of the host, the throughput is approximately constant. However, this constant value is far from the maximum value. This assurance is counterbalanced by a low throughput.

The best value for the window width (if it is to be fixed) seems to be ${ }^{*} N=\left(\sum_{i=1}^{K} M_{i}+\underset{i}{\inf }\left(M_{i}\right)\right) / 2$. In figures 9 and 10 this value corresponds to $\mathrm{N}=15$. In this case the maximum throughput (obtained for $\rho=1$ ) is intermediate between maximum throughput of RFC and XFC.

Performance does not seem to be very sensitive to this parameter setting. Besides means to regulate the value fo the window width are easier than those used to throttle the rate for the RFC policy.

The view of figures 9 and 10 gives an idea of an efficient dynamical WFC : the use of the upper envelop of the curves. For example on Figure 9 , as soon as $\rho<0.8, \mathrm{~N}=25$; if $0.8<\rho<0.9, \mathrm{~N}=20$; if $\rho>0.9$, $\mathrm{N}=15$. When a threshold is exceeded no admittance is allowed until the number of packets in the PSN is above the associated window width.

As a conclusion of this comparison of flow control policies, we have written on the side of figure 10 the throughput that can be reached by each of the three techniques described previously. We see that the zone corresponding to the RFC goes from the highest to the lowest point. WFC is intermediate. A very precise throughput is associated with XFC.

It has to be noted that the more we want to get high performance, the more the control of parameters must be sophisticated, otherwise the throughput will be very low.

\section{6 - CONCLUSIONS.}

First we have shown that a flow control is necessary in PSN's. Without flow control schemes a thrashing phenomenon occurs when the traffic rate reaches a value close to 1 . Several flow-control policies have been modeled and comparisons of the results can be interpreted as follows. The XFC technique allows one to obtain a certain throughput which does not decrease with increasing input traffic even when the system is saturated. We are sure that whatever the traffic conditions, * note : this value has been determined heuristically on a large number
of examples. 
a certain amount of service will always be rendered. However, the maximum throughput is very low in relation with the throughput obtained by other flow-control policies.

The RFC (rate flow contro1) policy gives efficient results when it is easy to find accurate rate limitation corresponding to the optimal throughput. If the network is well balances or very simple, such as a tandem queueing system, the value of the limitation can be obtained. But in a complex network, this rate limitation will have to change with the state of the network. A system of control packets must be created for the host to know the state of the system.

The RFC policy will allow us to obtain large throughput; but a necessary condition is the need to have a sophisticated control system. Thrashing can appear here with bad management.

The WFC policy can be considered as an intermediate method. The maximum throughput is not as high as in the previous scheme, but there is no thrashing in saturation conditions. Moreover, the simplicity of this scheme can be a good reason for its implementation.

Finally the question that can be raised is the accuracy of the previous results. Some validations by simulations of the unified mathematical model results have been done and are available in [9]. It is shown that even when the model parameters are somewhat different, the form of the curves is identical and the conclusions of the comparisons are similar.

A more widely available validation can be found in [10] where the predictions of a mathematical model (with the parameters used here) are compared to the results of a measurement compaign on the Cyclades network. The model being a queueing system in tandem, the very good accuracy of the mathematical model prediction is established. 


\section{REFERENCES}

[ 1] Davies D.W., "The control of congestion in packet-switched networks", IEEE Trans. on Communications 20, 3, 546-550, 1972.

[ 2] Price, "Simulation studies of an isarithmically controled store and forward data communications network", Proc. IFIP 74, Stockholm, $151-154,1974$.

[ 3] Pouzin L., "Cigale, the packet switching machine of the Cyclades computer network", Proc. of IFIP 74, Stockholm, 155-159, 1974. [ 4] CCITT "Recommendation X25", Orange Book, Vol. VIII.2, Geneva,
1977.

[ 5] Grangé J.L., Mussard P., "Performance Measurements of line control protocols in the Cigale Network", Computer Network, August 1978.

[ 6] Gelenbe E., Labetoulle J., Pujolle G., "Performance evaluation of the protocol HDLC" Computer Network, August 1978.

[ 7] Kleinrock L., "Communication nets-stochastic message flow and delay", McGraw-Hi11, New York, 1964.

[ 8] Pujolle G., "The influence of protocols on the stability conditions in packets-switching networks", IEEE Trans, on Communications, March 1979.

[ 9] Pujolle G. "Modélisation et évaluation de performances des réseaux à commutation de paquets", Thèse d'Etat, Université d'Orsay, 1978.

[10] Eyries F., Pujolle G., "Validation and prediction of performance in the Cigale network", Proc. of ICPCI 78, Gardone Riviera, Italy 1978.

[11] Gelenbe E. Pujolle G., "Approximation to a single queue in a network" Acta Informatica 7, 123-136, 1976. 


\section{APPENDIX}

Computation of the throughput as a function of the activity of the host

1. The model without station $\mathrm{C}$ and $\mathrm{R}$

We recall the assumptions we have made :

- the independence assumption

- the distributions of service times of all the stations are identical

- a customer leaving a queue sees the system at steady state.

Let $\mu_{n}^{-1}$ and $K s_{n}$ be the mean and the squared coefficient of variation (SCV) of the service time distribution of server $n$. We show in figure Al, the model that we want to study (we have only depicted stations $n$ and $n+1)$. We denote by $\lambda_{n}, K_{n}$ the rate and the SCV of interarrival distribution respectively to station $n$, (before rejection which occurs with probability $p_{n}$ ).

Analysis of the switch retransmission model.

We will replace each station (for example station $n$ ) with its retransmission loop, by a simple queue with a service time representing the time of the first retransmission plus all the retransmissions of the same packet. Let $\hat{\mu}_{n}$ and $\hat{K} s_{n}$ determine the distribution of this service time. Using a convolution product we obtain :

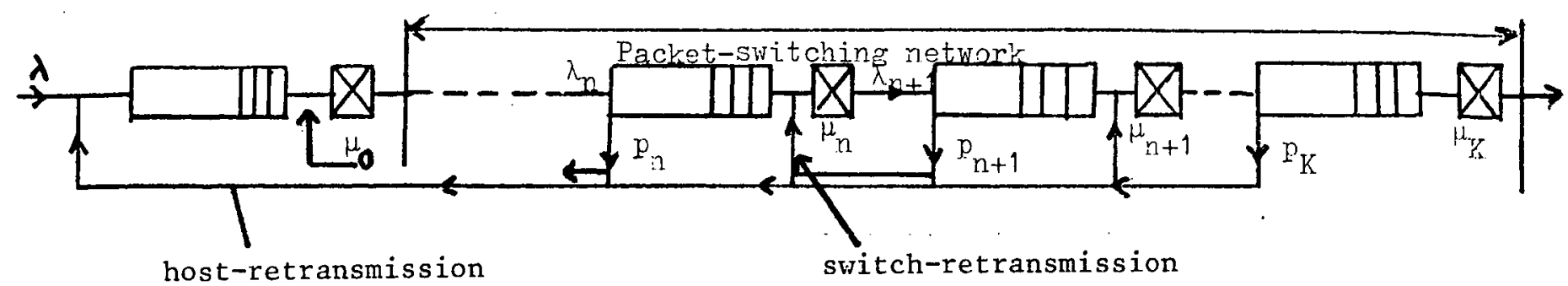

Figure A1

The tandem queueing model 


$$
\begin{aligned}
& \hat{\mu}_{n}=\mu_{n}\left(1-p_{n+1}\right) \\
& \hat{K}_{n}=p_{n+1}+K s_{n}\left(1-p_{n+1}\right)
\end{aligned}
$$

To compute the two first moments of the input flow we must include all transmissions and retransmissions from station $\mathrm{n}-1$; hence $\lambda_{n}=\lambda /\left(1-p_{n}\right)$ and $K a_{n}=-1+\rho_{n-1}^{2}\left(K s_{n-1}+1\right)+\left(2 \rho_{n-1}+1+K a_{n-1}\right)\left(1-\rho_{n-1}\right)$ where $\rho_{n}=\lambda_{n} / \hat{\mu}_{n}$. This last expression is a particular case of the SCV of the interarrival flow in a station computed in [11] from a general network.

Thus, each station $n$ is treated as a $G / G / 1 / M_{n}$ system with a service time distribution determined by $\hat{\mu}_{n}$ and $\hat{\mathrm{K}}_{\mathrm{n}}$ and an arrival time distribution by $\lambda_{\mathrm{n}}$ and $\mathrm{Ka}{ }_{\mathrm{n}}$.

The probability that a packet is refused at this station is computed through a diffusion approximation [8] : it is the probability at steady state that the queue is fu11

$$
p_{n}=\frac{\rho_{n}\left(1-\rho_{n}\right)}{e^{-\gamma_{n}\left(M_{n}-1\right)}-\rho_{n}^{2}}
$$

where $\ddot{\rho}_{n}=\lambda_{n} / \hat{\mu}_{n}, \quad \gamma_{n}=2 b_{n} / a_{n}, \quad a_{n}=\lambda_{n} K_{n}+\hat{\mu}_{n} \hat{K}_{n}$ and $b_{n}=\lambda_{n}-\hat{\mu}_{n}$.

Note that $p_{n}$ depends on $p_{n+1}$ by the intermediate of $\hat{\mu}_{n}$ and $\hat{K} s_{n}$. But if we begin to solve the equations for the last station first, we have $\mathrm{p}_{\mathrm{K}+1}=0$ and so we can compute, one after another, the values of $\mathrm{p}_{\mathrm{n}}$ for $n=K$ to $n=1$.

As we have assumed that the distributions of service times of al1 the stations are identical, we can suppose the mean service time equal to a time unit. We have to note also that $p_{1}$ is an increasing function of $\bar{\lambda}$ the normalized arrival rate (mean service time=1) such that the value of the activity of the host determines an unique value of the external arrival rate $\bar{\lambda}$. Therefore, for a given utilization $\rho$ of the 
host, by an iterative method we can compute $\bar{\lambda}$ and $p_{1}$ (for a given $\bar{\lambda}$ we get step by step $p_{n}, n=K$ to $n=1$, thus $p_{1}$; the exact value of $\bar{\lambda}$ is obtained when the equality $\rho=\frac{\lambda}{1-p_{1}}$ holds). Now the value of $S(\rho)$ is derived as :

$$
\begin{aligned}
& \mathrm{S}_{\mathrm{sr}}^{\mathrm{SW}}(\rho)=\left(\mathrm{CaL}+\mathrm{Cb}+\frac{\mathrm{CaL}}{2} \rho\right)\left(1-\mathrm{p}_{1}\right)+\mathrm{Tp}_{1} \\
& \mathrm{~S}_{\mathrm{hr}}^{\mathrm{HDLC}}(\rho)=\mathrm{CaL}\left(1-\mathrm{p}_{1}\right)+\mathrm{Tp}_{1}
\end{aligned}
$$

following the retransmission policy.

Therefore, the total arrival rate is $\lambda^{*}=\rho / S(\rho)$. As this rate is the sum of external arrivals and recycling packets, the throughput of the system is

$$
\lambda=\lambda^{*}\left(1-\mathrm{p}_{1}\right)
$$

Analysis of the host-retransmission scheme

The arrival rate at station $n+1$ is $\lambda_{n+1}=\lambda_{n}\left(1-p_{n}\right)$ for $n=1$ to $k-1$; if we conveniently denote by $\lambda_{\mathrm{K}+1}$ the departure rate from the last station, $\mathrm{K}$, then the preceding equation is also valid for $\mathrm{n}=\mathrm{K}$.

As we use the host-retransmission only with exponentially distributed service times, we develop the solution only in this particular case. An extension to general service times can be done through a diffusion approximation.

The probability of refusal is obtained through the classical $M / M / 1 / M_{n}$ queue :

$$
p_{n}=\rho_{n}^{M} \frac{1-\rho_{n}}{1-\rho_{n}} \text { where } \rho_{n}=\lambda_{n} / \mu_{n}
$$

We also know that the departure rate from the last station is equal to the external arrival rate into the network, i.e. $\lambda_{K+1}=\lambda$. Thus, beginning with the last station, we can compute $\left(\lambda_{n}, p_{n}\right)$, for $n=K$ to $n=1$. The host is a $M / M / 1$ system with service time $S(\rho)$ and arrival rate $\lambda^{\star}=\lambda+\sum_{i=1}^{\mathrm{K}} \mathrm{p}_{i} \lambda_{i}$. 
The service time $S(\rho)$ depends on the node-to-node protocol :

$$
\begin{aligned}
& S_{h r}^{S W}(\rho)=\mathrm{CaL}+\mathrm{Cb}+\frac{\mathrm{CaL}}{2} \\
& S_{h r}^{\mathrm{HDLC}}(\rho)=\mathrm{CaL}
\end{aligned}
$$

Therefora for a given utilization rate of the host we obtain the total arrival rate $\lambda^{*}=\rho / S(\rho)$, and the throughput of the system is obtained by an algorithmic method determining $\lambda$ when the following equality holds :

$$
\lambda=\lambda^{*}-\sum_{i=1}^{K} p_{i} \lambda_{i} .
$$

The solution is unique because the $p_{i}$ 's are increasing with $\lambda$.

2. The mode1 with stations $R$ and $C$.

The mode 1 has been shown in figure 3 . We are interested only by the switch-retransmission policy.

The solution we propose is to use an equivalent station. The closed network representing the model of the PSN itself can be replaced by a single queue with a state dependent rate $v(j), j=1, \ldots, N$ ( $N$ is the total number of credits, namely the total number of packets in the PSN plus the free credits). We have to study a closed queueing network with finite buffer size. As no analytical method is available to study such a queueing system, we adopt a simulation just to compute the utilization $A_{K}^{j}$ of the server $K$, when there are $j$ customers in the closed network. We have assumed in this simulation that the service time of the credit queue equals the host service time. The equivalent service rate is obtained as $v(j)=A_{K}^{j}$, assuming the mean service time of each station is a time unit.

The equivalent system is shown in figure A2 


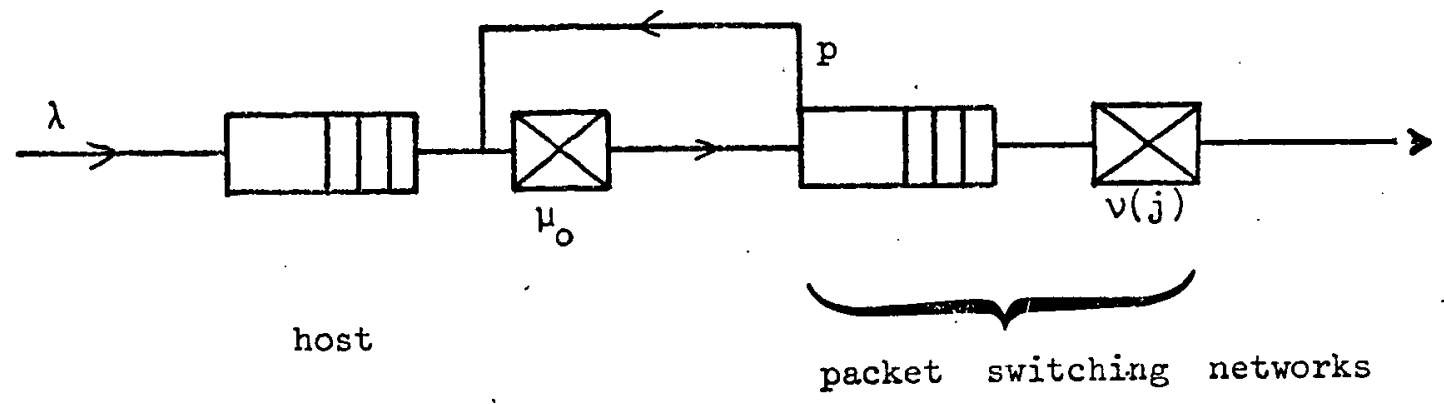

The equivalent unified model

Figure A2

The rejection probability $\mathrm{p}$ for a customer is always taken as the probability the second queue is full, so :

$$
p=\frac{\frac{\rho}{v(1) v(2) \ldots v(\mathbb{N})}}{1+\frac{\rho}{v(1)}+\ldots+\frac{\rho^{N}}{v(1) \ldots v(N)}}
$$

where $P$ is the utilization of the host.

Now the service time $S(\rho)$ can be computed and is equal to either $\mathrm{S}_{\mathrm{Sr}}^{\mathrm{SW}}$ or $\mathrm{S}_{\mathrm{sr}}^{\mathrm{HDCL}}$ following the node-to-node protocol.

The total arrival rate is $\lambda^{*}=\rho / S(\rho)$, and the throughput of the system is

$$
\lambda=\lambda^{*}(1-p) .
$$


